\title{
Accueillir la décentralisation en région : quelques réflexions sur un modèle de gouvernance territoriale en Outaouais
}

\author{
Martin Robitaille \\ Université du Québec en Outaouais
}

$\int$

a régularité avec laquelle le discours sur la décentralisation revient à l'ordre du jour des discussions politiques est assez déconcertante. À tel point que, pour plusieurs, cela relève davantage de l'opportunisme politique que de réelles intentions de l'État québécois. Il demeure que, d'une fois à l'autre, les régions se prennent au jeu de l'inéluctable nécessité de ne pas laisser passer leur chance et de réfléchir sur le pourquoi et le comment; sur le besoin et l'intérêt de revendiquer de nouvelles compétences et de nouveaux pouvoirs afin de mieux maîtriser leur propre développement. Cependant, un fait demeure, les régions affinent avec le temps leurs propositions et leurs revendications sur la décentralisation tout en se méfiant davantage des expériences antérieures. Un passé pas si lointain où la décentralisation n'avait comme résultat que de déléguer à des niveaux inférieurs (régions et municipalités) des responsabilités sans les ressources appropriées.

À l'automne 2002, le gouvernement du Parti québécois organisait le "Rendez-vous national des régions » afin de mieux comprendre les besoins des régions et de mieux répondre à leurs attentes. Cet événement, réclamé au départ par toutes les régions du Québec, se voulait une occasion de faire valoir une série de revendications portant sur le développement économique, l'autonomie des régions, la vitalité des milieux, particulièrement dans les régions ressources confrontées à des problèmes de décroissance démographique.

Pour ne pas être en reste, le nouveau gouvernement libéral organise à son tour, deux ans plus tard, sa consultation «Briller parmi les meilleurs » où l'on questionnait la population sur l'importance de réaliser la décentralisation, d'assurer le développement régional et rural pour rendre les régions plus prospères, d'encourager l'installation des nouveaux Québécois en région et d'améliorer la démocratie municipale.

Comme on le sait, ce n'était pas la première fois que les régions étaient appelées à ce type de consultation. Ces événements, même s’ils n’ont pas eu les retombées escomptées, ont permis de mobiliser les régions, de les solidariser davantage et, surtout, pour plusieurs d'entre elles, de faire avancer la réflexion sur la décentralisation.

Ce texte aura donc comme objectif de faire état des travaux menés en Outaouais sur la vaste question de la décentralisation régionale. Il s'agira de montrer comment cette région est arrivée à amorcer une réflexion de fond sur la décentralisation à partir d'un regard critique sur son propre fonctionnement afin de mieux s'approprier ses diverses dimensions tout en développant une structure régionale d'intervention plus adaptée à une gestion décentralisée des nouvelles compétences souhaitées. Nous verrons aussi comment les changements politiques et organisationnels issus de la loi 34 qui, entre autres, accorde aux élus locaux le rôle de décideurs en regard du développement local et régional au sein des CRÉ (Conférences régionales des élus) et au conseil d'administration des CLD (Centres locaux de développement), sont venus interférer dans la mise en place du modèle préconisé par la collectivité.

Auparavant, il serait judicieux de regarder comment cette dynamique régionale de concertation, qui a permis de structurer ce mode d'intervention régionale, s’inscrit dans le nouveau modèle québécois de développement. 


\section{Le modèle québécois de développement}

Force est d'admettre que, dans la dernière décennie, l'importante modification des structures régionales et locales de concertation et de services a permis aux régions de se doter d'une plus grande capacité de concertation et de réalisation d'actions structurantes sur leur territoire. Le travail de mobilisation et de planification des CRD (Conseils régionaux de développement) et la mise en place des CLD ont été des mesures concrètes permettant cette dynamisation des régions.

Sur le fond, c'est le développement d'un modèle québécois de gouvernance dont il est question ici et qui a inspiré une gouvernance particulière des régions. Un modèle partenarial ouvert à la société civile qui fait place à une gouvernance de collaboration entre l’État, le marché et la société civile ${ }^{1}$.

Pour les régions, le changement est important, particulièrement en ce qui a trait au rôle de l'État, qui a changé son intervention étatique de planification « par le haut » à une intervention arrimée territorialement et souvent en interface avec les élus, et à la société civile ${ }^{2}$. Il faut dire que cette volonté de faire du développement endogène sur les territoires a eu des effets majeurs sur le renforcement de la capacité interne de développement des régions. Non seulement pour les régions en voie de dévitalisation, mais aussi pour celles qui cherchent à intégrer la nouvelle économie dans leur développement.

La force du modèle québécois résiderait donc dans sa capacité d'accroître la concertation entre les acteurs et de développer de nouvelles formes de collaboration entre les entreprises, les institutions locales et les divers acteurs sociaux. Un modèle qui est aussi en mesure de mobiliser la société civile sur des enjeux importants de développement et de pouvoir mettre en place des mécanismes et des stratégies permettant d'agir en concertation avec plusieurs intervenants, qu'ils soient publics, communautaires, associatifs ou privés $^{3}$. Cependant, un effet non négligeable de l'essor du modèle québécois de développement a été un accroissement du désir d'autonomisation régionale et locale au Québec. Certes, plusieurs années de pratiques concrètes dans la gestion territoriale ont permis de mieux réaliser à quel point la capacité des acteurs locaux (société civile et élus) à se prendre en main s'est grandement renforcée.

\section{La force du modèle québécois résiderait donc dans sa capacité d'accroître la concertation entre les acteurs et de développer de nouvelles formes de collaboration entre les entreprises, les institutions locales et les divers acteurs sociaux.}

\section{La décentralisation : réflexions sur la gouvernance en Outaouais}

Lors du «Rendez-vous des régions » de novembre 2002, le gouvernement a invité les régions du Québec à déposer des projets visant le développement de leur région. La délégation de l'Outaouais fut la seule à ne déposer qu'une demande en matière de compétences, soit les pouvoirs de décider, d'agir et de dépenser dans tous les secteurs sociaux et économiques qui ont un impact sur le développement de la région. Cette demande a soulevé, dans un premier temps, l'ire du gouvernement puisqu'elle allait bien au-delà de ses anticipations touchant la décentralisation. D'autant plus que cette région a du coup réclamé un transfert des juridictions et des ressources financières appropriées pour la prise en charge du développement régional, notamment dans la mise en œuvre des cibles de l'Entente-cadre entre l'Outaouais et Québec. En toile de fond à cette demande se retrouve une réforme fiscale et administrative dont l'objectif visait un transfert des points d'impôt, des redevances sur ses ressources naturelles et l'application d'une péréquation pour doter la région d'un contrôle accru sur son avenir socio-économique. Dans les faits, il s'agissait de reconnaître que l'Outaouais était en mesure de s'attaquer efficacement aux problèmes qui freinent son développement, que ce soit en matière d'éducation, de problèmes sociaux, de développement économique ou d'infrastructures. Sur cette base, la région tentait de démontrer qu'une véritable décentralisation des pouvoirs vers une "autorité régionale imputable » doit s'accompagner des réformes essentielles qui permettent d'affecter efficacement et intelligemment les fonds publics. En conclusion des travaux de cet événement, l'Outaouais s'était vu confier le mandat de développer un modèle de décentralisation régionale ${ }^{4}$.

C'est dans un contexte politique changeant (élection du gouvernement libéral) et d'une modification substantielle des structures de développement régional 
(mise en place des CRÉ et gestion des CLD par les MRC) qu'un groupe de travail a analysé les modèles de décentralisation et développé une structure d'intervention mieux adaptée à un éventuel processus de décentralisation régionale. Il faut dire cependant que l'exercice a nécessité une analyse critique et sans complaisance des vingt dernières années de concertation régionale dans la région et a mobilisé beaucoup d'intervenants ${ }^{5}$.

\section{Les limites du modèle antérieur de la concertation régionale en Outaouais}

Après des années de consultations régionales et locales dans plusieurs secteurs, d'exercices de planification stratégique régionale (PSR), de plans d'action locale pour l'économie et l'emploi (PALÉE), d'ententes spécifiques avec le gouvernement, l'expertise territoriale en matière de consultation et de concertation s'est considérablement développée en Outaouais. Après analyse, le groupe de travail a fait un certain nombre de constats qui ont le mérite de montrer que, même avec beaucoup de volonté, la région de l'Outaouais aurait besoin de mieux se structurer pour accueillir toute forme de décentralisation. Une restructuration qui touche le cœur du dynamisme de la région, soit le processus de concertation régionale.

Ces principaux constats soutiennent que la concertation régionale aurait atteint ses limites et cela, pour plusieurs raisons. D'abord parce que l'inadéquation entre le système actuel et la réalité régionale est trop forte. De fait, la réalité contemporaine des régions est plus complexe qu'elle l'était il y a vingt ans. Il est sans contredit beaucoup plus difficile d'intervenir efficacement en région lorsque tout est décidé sans considération des particularités régionales. Les structures gouvernementales actuelles répondent mal aux besoins exprimés par la région et provoquent de vives tensions entre les visions nationales et les perceptions locales.

Ce problème se reflète bien par le fait d'une administration gouvernementale régionale beaucoup trop centralisée. Au point où ce serait une vision nationale qui caractérise l'organisation et l'action gouvernementale sur le territoire, ce qui, dans les faits, rendrait bien difficile l'adaptation des stratégies et des actions aux besoins de la région. Certes, la conférence administrative régionale (CAR) a comme fonction de coor- donner l'action gouvernementale en Outaouais, mais cette dernière est aussi tributaire des tensions et des volontés ministérielles centralisatrices. Ainsi, la tension « centre-région » de même que l'absence de certains ministères et organismes dans plusieurs régions du Québec demeurent des irritants majeurs à toutes formes de décentralisation.

Outre ces tensions, il importe de souligner la trop grande distance entre le politique et l'administratif, ce qui a comme conséquence directe de freiner l'action en région. Ainsi, au cours des années et avec des directives politiques claires, plusieurs ministères ont contribué, chacun à leur façon et sans trop de consultations territoriales, à la création et au financement d'organismes sectoriels ayant des mandats d'intervention au niveau local ou même régional. Certes, ces organismes leur ont permis d'atteindre des objectifs ministériels nationaux. Cependant, ces organisations sont essentiellement tributaires des orientations gouvernementales véhiculées par les directions régionales de ces ministères. Il s'agit donc d'une relation du haut vers le bas sous forme de "silos » permettant trop souvent d'asseoir les volontés gouvernementales en région. Ce type d'intervention a eu pour principal effet une multiplication des structures sectorielles en région. Qui plus est, le gouvernement, en conservant dans plusieurs cas, des liens directs avec ces organismes - et en l'absence d'autorité régionale - a fait en sorte de provoquer une multiplication des organisations, des lieux de décisions et des actions sur un territoire, ce qui augmente d'autant la difficulté de dégager, dans la collectivité, une vision d'ensemble du développement territorial.

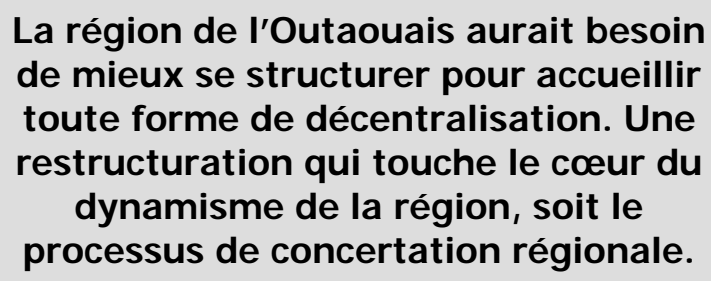

Cette multiplicité d'organismes, avec des missions et des mandats souvent très pointus, ne favorise pas nécessairement la concertation. Néanmoins, la pertinence et la mission de ces organisations économiques, sociales et communautaires sur le territoire ne sont pas à remettre en cause. Seulement, force est de constater que le fait d'intervenir sur un territoire donné 
(local ou régional), sur un secteur d'activités précis (santé, éducation, entrepreneuriat) ou encore sur des clientèles spécifiques (jeunes, femmes, immigrants, etc.) cause une juxtaposition des activités des organismes et est une source de tensions. Cela demeure particulièrement vrai quand les ressources financières se font rares.

Dans les faits, c'est la capacité de mobilisation et de concertation régionale qui serait en danger. À preuve, pour ce comité de travail, les quelque 18 tables de concertation locale, régionale, et sectorielle qui ont vu le jour depuis le début des années 1990. Pourtant si ces tables existent, c’est par la nécessité de dégager une vision commune et d'agir collectivement en fonction des besoins de la région et parce que les organisations ne peuvent plus répondre seules aux problèmes auxquels leurs territoires, leurs secteurs d'intervention ou leurs clientèles sont confrontés. Or, l'éventail grandissant de problèmes régionaux requiert une mobilisation d'envergure qui ne saurait être qu'une superposition de comités et de structures engendrant le symptôme des «TLM » (toujours les mêmes). Plus encore, c'est la capacité de ces multiples structures de consultation et de concertation de dégager des stratégies et des actions de façon intersectorielle qui fait grandement défaut.

Les limites actuelles du modèle de concertation en Outaouais ont été atteintes car, même après la mise en place d'une concertation locale et régionale sans précédent, dans les faits les résultats escomptés se font attendre. Il y a perte d'efficacité et d'efficience dans l'action parce que les mêmes acteurs se retrouvent partout. Dans une perspective de décentralisation, le défi demeure de trouver un fonctionnement qui permette une certaine rationalisation des activités non plus en fonction des besoins ministériels ou organisationnels, mais d'une volonté régionale d'offre de services dans le but de créer une meilleure cohésion et de réaliser des économies d'échelle en termes de ressources et de services.

\section{Proposition d'une structure régionale d'intervention}

Dans sa réflexion amorcée en janvier 2003, ce comité a travaillé à modéliser une structure d'intervention qui favoriserait une réflexion régionale et qui donnerait du sens aux projets et aux actions initiées par la région. Il s’agissait de concevoir une structure qui re- présente davantage un lieu où pourront être partagées non seulement une vision commune du développement de la région, mais aussi une manière générale de la réaliser. Ce lieu doit aussi être en mesure de soutenir la mobilisation afin d'amener les décideurs, les intervenants et la population à s'investir de façon continue dans la construction de partenariats.

Le modèle proposé en a été l'objet d'une consultation régionale en octobre 2004 qui regroupait plus de 200 intervenants régionaux et élus. Ce modèle repose essentiellement sur la création de cinq commissions régionales (la commission Santé, Services sociaux et Développement social; la commission Éducation, Recherche et Science; la commission Culture et Loisirs; la commission Développement économique, Emploi et Tourisme; la commission Aménagement du territoire et Développement durable) et d'une table intersectorielle (voir la figure 1). Chacune des commissions régionales se veut un lieu de planification, de recommandation, de mise en œuvre et d'évaluation des actions structurantes dans des champs de compétences spécifiques. Elles devraient permettre d'établir une communication continue entre la Conférence régionale des élus de l'Outaouais (CRÉO) et les milieux sectoriels régionaux.

- La commission Santé, Services sociaux et Développement social vise notamment les enjeux liés à la santé et aux services sociaux, à la jeunesse, au vieillissement de la population, à l'immigration, à la famille, à la sécurité sociale, au logement social, à la lutte contre la pauvreté, à l’équité...

- La commission Éducation, Recherche et Science concerne entre autres les enjeux liés à l'éducation primaire et secondaire, à la formation professionnelle, à l'enseignement collégial et universitaire, à la recherche, à la science, à l'innovation et au transfert technologique...

- La commission Culture et Loisirs vise principalement les enjeux liés à la culture, aux loisirs scientifiques, culturels et sportifs...

- La commission Développement économique, Emploi et Tourisme s’intéresse particulièrement aux enjeux liés à l'emploi, à l'entrepreneuriat, au support au développement (financement et servicesconseils), au développement des secteurs économiques prioritaires (tourisme, technologie, forêt, agroalimentaire)... 
- La commission Aménagement du territoire et Développement durable vise surtout les enjeux liés à l'aménagement du territoire, le développement des infrastructures (transport, communication...), l'environnement, la gestion de l'eau, des matières résiduelles, la gestion par bassins versants, l'élaboration des plans de développement intégré (PDI)...

Les mandats des commissions régionales sont principalement d'assurer une participation du citoyen et l'implication de la société civile dans le développement socio-économique local et régional tout en favorisant la concertation régionale. Elles ont à définir les problématiques, établir des priorités, élaborer des stratégies, fixer des objectifs régionaux, mettre en œuvre le plan quinquennal. Ces commissions doivent aussi viser l'intégration de préoccupations territoriales, sectorielles et transversales (jeunesse, vieillissement de la population, immigration, famille, sécurité sociale, logement social, lutte contre la pauvreté, équité, égalité et parité des femmes...). L’élaboration des critères d'évaluation et de répartition des ressources du secteur et la recommandation, au niveau décisionnel, de l'allocation des ressources et de l'organisation des structures régionales et locales de support, sont au cœur des préoccupations de commissions.

Figure 1 - Structure d'intervention régionale

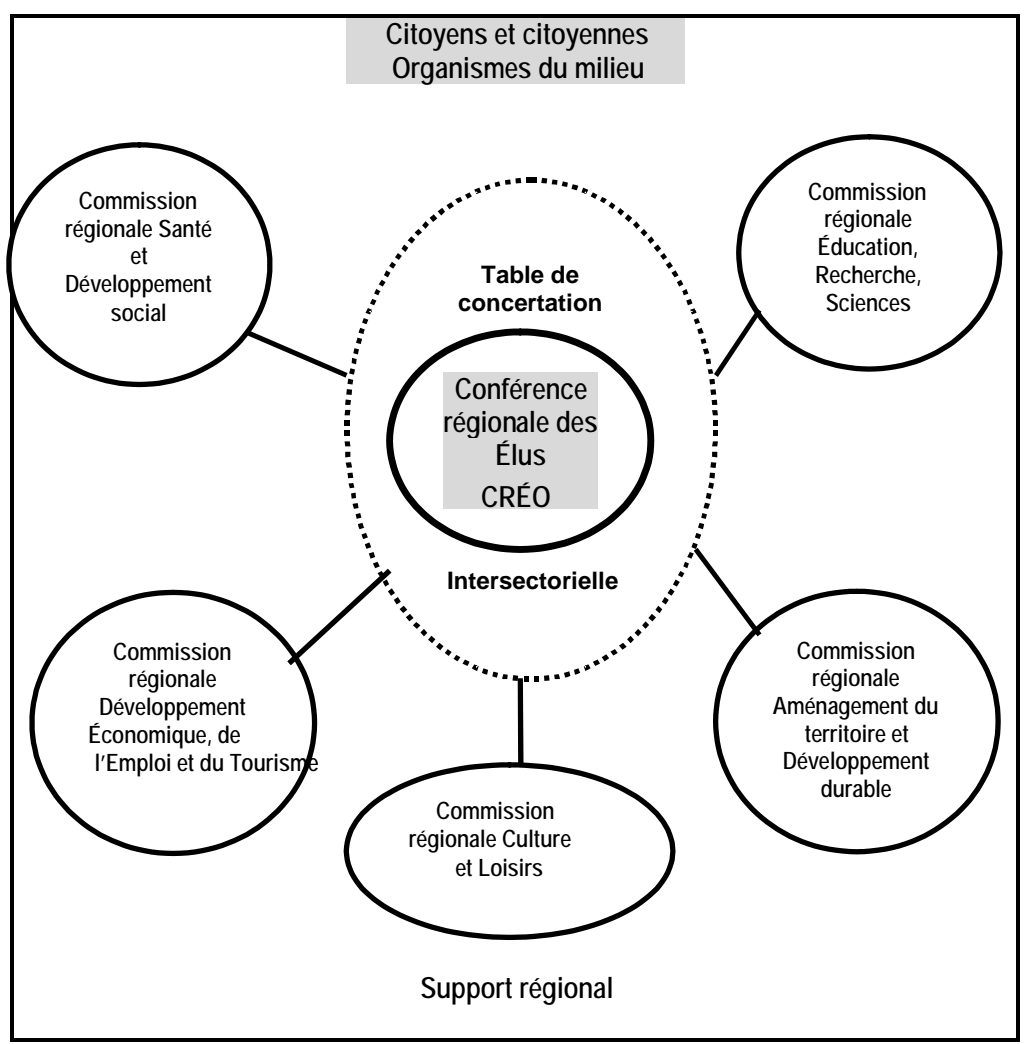

Par souci d'une certaine appropriation par les membres de la nouvelle Conférence régionale des élus de l'Outaouais, le comité de travail a laissé le soin à cette dernière de déterminer les modalités de nomination des membres des cinq commissions régionales. En l'occurrence, il était suggéré que les membres pourraient être choisis après consultation des organismes de divers milieux ou encore, la CRÉO pourrait instituer, avec les groupes socio-économiques de la région, un mécanisme de concertation établi sur une base sectorielle, thématique ou territoriale. Ce faisant, chacune des commissions serait représentée au conseil d'administration de la CRÉO.

Afin de pallier les lacunes du modèle précédent, en ce qui a trait au manque de coordination entre les tables sectorielles, le comité de travail a proposé la mise sur pied d'une table de concertation intersectorielle régionale. Cette dernière permet d'assurer la concertation et l'harmonisation intersectorielle régionale en vue de promouvoir une vision intégrée et cohérente de l'action des gouvernements, des institutions et organismes 
de support au développement régional et local dans ses dimensions économiques, sociales et culturelles. Elle se veut le lieu d'élaboration et d'évaluation du plan annuel et quinquennal de développement de l'Outaouais. À cette fin, cette table peut se doter de comités consultatifs permettant de mieux intégrer les problématiques de clientèles, notamment pour les jeunes et les femmes. Sa composition repose majoritairement sur des représentants des commissions régionales et des directions régionales des ministères du gouvernement du Québec qui sont concernés. La CRÉO en nommerait les membres en tenant compte d'une représentativité sectorielle, thématique ou territoriale.

\section{L'incertitude à l'égard des politiques de développement régional du gouvernement libéral et le temps requis pour la mise en place des nouvelles structures issues de la loi 34 (la mise en place de la CRÉO) ont eu plusieurs conséquences négatives sur le processus de concertation.}

Les mandats de la table de concertation intersectorielle régionale sont d'assurer, de définir et de promouvoir une vision du développement socio-économique régional qui intègre les orientations sectorielles des commissions, ses dimensions intersectorielles ainsi que transversales, notamment la santé, la jeunesse, le vieillissement de la population, l'immigration, la famille, le logement social, la lutte contre la pauvreté, l'équité, l'égalité et la parité des femmes, etc. Cette table vise aussi l'élaboration d'une planification stratégique régionale qui décrit les enjeux propres à la région en faisant état de ses forces et faiblesses tout en permettant d'identifier les axes et les priorités de développement de la région pour un horizon de cinq ans. Elle proposera l'adoption à la CRÉO du plan stratégique régional quinquennal.

Pour les membres du comité de travail, ce projet doit soutenir cinq principes qui seront la base essentielle de sa réussite. "Ils représentent le sens que nous désirons donner à cette volonté de décentralisation, une décentralisation qui se veut responsable, inclusive et portée vers l'avenir ». Les principes qui viendront soutenir cette vision sont la subsidiarité, l'implication de la société civile et la participation des citoyens, l'équité, l’imputabilité régionale et le développement durable.

\section{Les conférences régionales des élus : nouvelle donne de la gouvernance régionale}

Pendant la réflexion de ce groupe de travail, des changements importants dans la structuration du développement local et régional sont survenus. Dans les faits, la loi 34 instituant le ministère du Développement économique et régional et de la Recherche, qui crée pour chaque région administrative du Québec une "Conférence régionale des élus » et confie la gestion des CLD au MRC, est non seulement venue rompre avec le modèle québécois basé sur la participation de la société civile, mais a obligé le comité à prendre en considération cette nouvelle donne. Dans ce nouveau contexte, l'adaptation de la structure régionale d'intervention en Outaouais est apparue primordiale afin de pallier en partie les conséquences de ces changements dans le mode de gouvernance du développement régional et local. Surtout que derrière ce changement se retrouvait une volonté explicite du gouvernement libéral d'effectuer une décentralisation en régions de nouvelles compétences.

En Outaouais, l'incertitude à l'égard des politiques de développement régional du gouvernement libéral et le temps requis pour la mise en place des nouvelles structures issues de la loi 34 (la mise en place de la CRÉO) ont eu plusieurs conséquences négatives sur le processus de concertation institué depuis plus de deux décennies dans la région. Ces changements ont eu comme suite immédiate de paralyser toute activité de consultation et de concertation dans la région pendant près de deux ans. Rappelons que, même si la première rencontre officielle des membres de la CRÉO s'est déroulée quelques semaines après l'adoption de la loi 34, le temps requis pour amener les membres à s'entendre sur la composition définitive de la CRÉO, à adapter leur rôle d'élus locaux à gestion régionale et à assimiler le fonctionnement de l'organisation régionale a fait en sorte qu'il s'est écoulé près de 18 mois entre la création de la CRÉO et l'établissement d'un nouveau mode de concertation régionale en $\mathrm{Ou}-$ taouais.

Les effets sur la mobilisation de la collectivité en regard des actions entreprises dans le cadre de la planification stratégique 2000-2005 ont été impor- 
tants, à tel point qu'il sera difficile, selon plusieurs acteurs concernés, de relancer un autre exercice du genre. Qui plus est, ces changements ont retardé la mise en place du modèle de concertation proposé et entériné par la population en octobre 2004. Finalement, cette « appropriation » des mécanismes de concertation régionale, par les élus, a transformé considérablement le modèle initial.

Ainsi, trois changements majeurs sont venus, selon nous, altérer le modèle au point de lui enlever beaucoup de crédibilité aux yeux de la collectivité. Premièrement, la représentation paritaire de la société civile et des élus au sein de la direction des commissions a été ignorée dans la nouvelle structure. Certaines commissions seront donc dirigées par un représentant de la société civile et d'autres par des élus. De plus, le nombre de personnes siégeant sur chacune des commissions a été ramené à huit, ce qui est nettement moins que ce que proposait le modèle initial qui se voulait davantage intégrateur des dynamiques et des problématiques vécues dans les champs de compétences des commissions régionales. Autre changement majeur, la table de concertation intersectorielle a été abolie. Pourtant, elle représentait l'élément clé du modèle, car elle devait répondre aux faiblesses du système antérieur en permettant une intégration non seulement sectorielle et intersectorielle du travail des commissions, mais aussi un meilleur arrimage des dimensions transversales des problématiques soulevées comme celles des jeunes, de l'égalité et de la parité des femmes, du vieillissement de la population, de l'immigration, de la lutte contre la pauvreté, etc. Des dimensions importantes qui ne peuvent pas être considérées dans leur ensemble dans le cadre des commissions sectorielles. C'est d'ailleurs une des principales raisons qui avaient été à l'origine de la création de cette table intersectorielle.

Deux motivations semblent avoir été à la source des modifications du modèle entériné par la communauté. Une première, pragmatique, qui est liée à une volonté de simplifier l'exercice afin de diminuer le temps requis par les élus pour siéger à ces commissions et à la table intersectorielle. Il faut dire que la volonté du gouvernement, lors de la mise en place des CRÉ, de ne pas rémunérer les élus locaux pour le temps consacré à cette fonction n'aide pas la contribution de ces derniers au développement régional. Dans les faits, l'investissement dans leur travail d'élus se fait d'abord au niveau municipal, là où leur fonction est re- connue. Le développement régional est de ce fait vu comme une obligation avec laquelle il faut composer. Une seconde intention, moins explicite, est de s'assurer un certain contrôle sur les finalités du processus en choisissant les membres des commissions et en impliquant le moins possible de personnes dans le processus de concertation. Même si les décisions sans appel reviennent aux membres de la CRÉO, en majorité des élus locaux, il n'en demeure pas moins qu'en agissant ainsi, ils se donnent un plus grand contrôle de la concertation régionale dans la région. Ce qui semble aller de pair avec la volonté explicite de la loi 34 qui est de donner les pouvoirs du développement local et régional aux élus locaux.

\section{Cette « appropriation » des mécanismes de concertation régionale, par les élus, a transformé considérablement le modèle initial.}

\section{Conclusion}

Loin d'être parfait, le modèle initial permettait une certaine souplesse et une meilleure intégration des problématiques sectorielles sur le territoire. De plus, la proposition initiale permettait d'apporter des réponses aux problèmes soulevés par le modèle antérieur de concertation. D'une part, parce qu'il redonnait une plus grande place et une meilleure légitimité aux représentants de la société civile à la CRÉO. D’autre part, parce que ce modèle permettait d'identifier et de renforcer la prise en compte des dimensions multisectorielles du développement local et régional. Ce modèle aurait permis de créer de l'horizontalité là où il y a en ce moment de la verticalité par la mise en valeur de potentialités régionales et de leurs synergies correspondantes. Il s'agit, entre autres, de développer une plus grande synergie entre les acteurs du développement au sein d'un secteur (passage de 18 tables sectorielles à 5 commissions) dans la réalisation de leurs actions et, par là, de favoriser une meilleure intégration des problématiques sectorielles tout en permettant un regroupement des ressources autour d'une stratégie plus globale de développement. Du reste, la capacité de cette structure régionale d'intervention à orienter les élus locaux dans la détermination des priorités et des actions dans le milieu et de créer des consensus entre la société civile et les élus n'était pas négligeable. 
L'avenir nous dira comment la collectivité réagira avec le modèle modifié par les membres de la CRÉO. Cependant, le temps et l'exercice concret des commissions risquent fort de ramener sur la table le modèle proposé initialement car, dans le contexte actuel, ce sont les voix de plusieurs clientèles (jeunes, femmes, immigrants, etc.) qui ne seront pas assez entendues lors de l'exercice de planification stratégique 2005-2010.

Certes, nous ne connaissons pas encore comment le gouvernement entend concrétiser cette volonté bien affichée de décentralisation, mais il n'en demeure pas moins qu'il devient nécessaire pour les régions de réfléchir et de trouver le modèle de gestion de nouvelles compétences le mieux adapté à leur réalité pour réagir rapidement aux changements que la décentralisation peut supposer.

\section{Notes et références}

1 Carrier, M. et S. Côté (2000), Gouvernance et territoires ruraux. Éléments d'un débat sur la responsabilité du développement, Québec, Presses de l’Université du Québec.
2 Bourque, G.L. (2000), Le modèle québécois de développement : de l'émergence au renouvellement, Québec, Presses de l’Université du Québec.

3 Lévesque, B. (2005), « Le modèle québécois de développement régional et local : vers le néolibéralisme et la fin du modèle québécois ? », dans D. Lafontaine et B. Jean (dir.), Territoires et fonctions. Tome 1 - Des politiques aux théories : le modèle de développement régional et de gouvernance en débats, Rimouski, Éditions GRIDEQ et Éditions du CRDT, p. 15-43.

4 Deux autres régions se sont vues confier un tel mandat, soit la Beauce-Appalaches et la Mauricie.

5 Ce groupe de travail mis sur pied par la délégation de l'Outaouais au Sommet des régions a convenu de réfléchir sur les mécanismes nécessaires pour « accueillir » et gérer de nouvelles compétences issues d'une décentralisation; il était composé de 27 personnes provenant de divers milieux (élus, représentants de la société civile, des ministères régionaux, du milieu de l'éducation, etc.) et a remis son rapport intitulé : « Décentralisation du pouvoir décisionnel et modernisation des structures d'intervention en matière de développement régional » en janvier 2004. 